\title{
THE NUMBER OF BINOMIAL COEFFICIENTS DIVISIBLE BY A FIXED POWER OF 2
}

\author{
F. T. HOWARD
}

Abstract. Define $f(n, j)$ as the number of binomial coefficients $\left(\begin{array}{c}n \\ r\end{array}\right)$ divisible by exactly $2^{j}$. We find formulas for $f(n, j)$ for $0 \leqq j \leqq 4$ and evaluate $f(n, j)$ for special values of $n$.

1. Introduction. In [1] L. Carlitz considered the problem of determining the number of binomial coefficients $\left(\begin{array}{l}n \\ r\end{array}\right)(r=0,1, \cdots, n)$ divisible by a fixed power of a prime $p$. In this paper we consider the same problem for $p=2$. We note that this problem is also discussed briefly in [3].

Let $n$ be a positive integer and define $f(n, j)$ as the number of binomial coefficients $\left(\begin{array}{c}n \\ r\end{array}\right)(r=0,1, \cdots, n)$ divisible by exactly $2^{j}$. We shall find formulas for $f(n, j)$ for $j=0, \cdots, 4$, and we shall evaluate $f(n, j)$ for special values of $n$. Several of the results of [1] for $p=2$ are generalized in this paper.

Throughout this paper we shall assume that

$$
n=2^{e_{1}}+2^{e_{2}}+\cdots+2^{e_{m}}, \quad 0 \leqq e_{1}<e_{2}<\cdots<e_{m} .
$$

We shall also use the following notation:

$e_{1}-e_{0}=k$ means $e_{1}=k-1$,

$e_{1}-e_{0}>k$ means $e_{1}>k-1$.

We also define any binomial coefficient $\left(\begin{array}{l}x \\ y\end{array}\right)$ to be 0 if $x$ or $y$ is negative or if $x<y$.

2. Evaluation of $f(n, j)$. If $n$ satisfies (1.1) and

$$
\begin{array}{rlrl}
r & =2^{b_{1}}+\cdots+2^{b_{v}}, & & 0 \leqq b_{1}<\cdots<b_{v}, \\
n-r & =2^{c_{1}}+\cdots+2^{c_{w}}, & 0 \leqq c_{1}<\cdots<c_{w},
\end{array}
$$

then it is well known that the exponent of the highest power of 2 dividing $\left(\begin{array}{c}n \\ r\end{array}\right)$ is $v+w-m[2$, p. 263]. It is clear, then, that for $j>0$,

$$
f(n, j)=a_{j 1} 2^{m+j-2}+a_{j 2} 2^{m+j-4}+\cdots+a_{j j} 2^{m+j-2 j}
$$

where $a_{j i}$ is the number of integers $r$ such that (2.1) holds, $v+w=m$ $+j$, and there are a total of $m+j-2 i \quad b_{h}$ 's and $c_{k}$ 's such that

$$
b_{h} \neq c_{s} \quad(s=1, \cdots, w), \quad c_{k} \neq b_{s} \quad(s=1, \cdots, v) .
$$

Received by the editors August 19, 1970.

AMS 1969 subject classifications. Primary 0505, 0510.

Key words and phrases. Binomial coefficient, composition of a positive integer. 
In other words $a_{j i}$ is the number of possible ways of doing the following: Select $i$ elements from $e_{1}, \cdots, e_{m}$. Call them $d_{1}, \cdots, d_{i}$ and for $k=1, \cdots, i$ write

$$
2^{d_{k}}=2^{d_{k-g(k)}}+\sum_{s=1}^{g(k)} 2^{\left(d_{k-8}\right)}
$$

where $g(k)$ is a positive integer $\leqq d_{k}$ and

$$
\sum_{k=1}^{i} g(k)=j \text {. }
$$

Let $\left\{x_{1}, \cdots, x_{m-i}\right\}=\left\{e_{1}, \cdots, e_{m}\right\}-\left\{d_{1}, \cdots, d_{i}\right\}$ and divide the $m+j$ numbers

$$
\begin{aligned}
x_{1}, \cdots, x_{m-i}, d_{1}-1, \cdots, d_{1}-g(1), \\
\quad d_{1}-g(1), \cdots, d_{i}-1, \cdots, d_{i}-g(i), d_{i}-g(i)
\end{aligned}
$$

into two sets $\left\{b_{1}, \cdots, b_{v}\right\}$ and $\left\{c_{1}, \cdots, c_{v}\right\}$ such that $0 \leqq b_{1}$ $\cdots<b_{v}, 0 \leqq c_{1}<\cdots<c_{w}$ and $v+w=m+j$.

We can use this idea to evaluate $f(n, r)$. Suppose (1.1) holds and

$$
\begin{array}{r}
e_{i}-e_{i-1}>s \text { for } q_{s} \text { terms } e_{i}(s=1, \cdots, 4) ; \\
e_{i}-e_{i-1}=1 \text { and } e_{i-1}-e_{i-2}>1 \text { for } q_{5} \text { terms } e_{i}, \\
=2 \text { for } q_{6} \text { terms } e_{i}, \\
>2 \text { for } q_{7} \text { terms } e_{i}, \\
>3 \text { for } q_{8} \text { terms } e_{i} ; \\
e_{i}-e_{i-1}=2 \text { and } e_{i-1}-e_{i-2}>1 \text { for } q_{9} \text { terms } e_{i}, \\
>2 \text { for } q_{10} \text { terms } e_{i} ; \\
e_{i}-e_{i-1}=1, e_{i-1}-e_{i-2}=1 \text { and } e_{i-2}-e_{i-3}>1 \text { for } q_{11} \text { terms } e_{i}, \\
>2 \text { for } q_{12} \text { terms } e_{i} ; \\
e_{i}-e_{i-1}=1, e_{i-1}-e_{i-2}=2, e_{i-2}-e_{i-3}>1 \text { for } q_{13} \text { terms } e_{i} ; \\
e_{i}-e_{i-1}=2, e_{i-1}-e_{i-2}=1, e_{i-2}-e_{i-3}>1 \text { for } q_{14} \text { terms } e_{i} ; \\
e_{i}-e_{i-1}=1, e_{i-1}-e_{i-2}=1, e_{i-2}-e_{i-3}=1,
\end{array}
$$

Then we have

$$
\begin{gathered}
f(n, 0)=2^{m}, \\
f(n, 1)=q_{1} 2^{m-1},
\end{gathered}
$$




$$
\begin{gathered}
f(n, 2)=q_{2} 2^{m}+\left(q_{5}+\left(\begin{array}{l}
q_{1} \\
2
\end{array}\right)\right) 2^{m-2} \\
f(n, 3)=q_{3} 2^{m+1}+\left(q_{2}\left(q_{1}-1\right)+q_{9}+q_{7}\right) 2^{m-1} \\
+\left(q_{11}+q_{5}\left(q_{1}-1\right)+\left(\begin{array}{l}
q_{1} \\
3
\end{array}\right)\right) 2^{m-3} \\
f(n, 4)=q_{4} 2^{m+2}+\left(q_{3}\left(q_{1}-1\right)+\left(\begin{array}{l}
q_{2} \\
2
\end{array}\right)+q_{8}+q_{10}+q_{16}\right) 2^{m} \\
+\left(q_{2}\left(\begin{array}{c}
q_{1}-1 \\
2
\end{array}\right)+q_{12}+q_{14}+q_{7}\left(q_{1}-1\right)\right. \\
\left.+q_{9}\left(q_{1}-2\right)+q_{13}+q_{6} q_{2}+q_{7}\left(q_{2}-1\right)\right)^{2^{m-2}} \\
+\left(\left(\begin{array}{c}
q_{1} \\
4
\end{array}\right)+q_{15}+\left(\begin{array}{c}
q_{5} \\
2
\end{array}\right)+q_{5}\left(\begin{array}{c}
q_{1}-1 \\
2
\end{array}\right)\right.
\end{gathered}
$$

For example, if $n=2^{4}+2^{5}+2^{20}+2^{26}+2^{28}$ we have $q_{1}=4, q_{2}=3$, $q_{8}=3, q_{4}=3, q_{5}=1, q_{7}=1, q_{8}=1, q_{9}=1, q_{10}=1$, all other $q^{\prime} \mathrm{s}=0$, and therefore

$$
\begin{gathered}
f(n, 0)=2^{5}, \quad f(n, 1)=4 \cdot 2^{4} \\
f(n, 2)=3 \cdot 2^{5}+7 \cdot 2^{3}, \quad f(n, 3)=3 \cdot 2^{6}+11 \cdot 2^{4}+7 \cdot 2^{2}, \\
f(n, 4)=3 \cdot 2^{7}+14 \cdot 2^{5}+16 \cdot 2^{3}+4 \cdot 2 .
\end{gathered}
$$

We note that the formulas for $f(n, 0)$ and $f(n, 1)$ can be found in [1] and [3].

3. Some special evaluations. Carlitz points out in [1] that if $e_{i}-e_{i-1}=1$ for $i=1, \cdots, m$ then $f(n, j)=0$ for $j>0$. Some of the other results of [1] are special cases of the following theorems, which we prove by using the method of $\$ 2$.

THEOREM 3.1. If (1.1) holds, $e_{i}-e_{i-1}=1$ for $m-1$ terms $e_{i}$ and $e_{r+1}-e_{r}=t+1, t>0$, for some $r \geqq 0$, and $m-r \leqq t$, then

$$
\begin{aligned}
& f(n, 0)=2^{m}, \\
& f(n, j)=2^{m-j}+2^{m-j+2}+\cdots+2^{m-j+2(j-1)} \\
& \quad(j=1, \cdots, m-r),
\end{aligned}
$$




$$
\begin{aligned}
& f(n, j)=2^{m+j-2}+2^{m+j-4}+\cdots+2^{m+j-2(m-r)} \\
& (j=m-r+1, \cdots, t) \text {, } \\
& f(n, t+m-r-j)=2^{r+t+j-2}+2^{r+t+j-4}+\cdots+2^{r+t+j-2 j} \\
& (j=1, \cdots, m-r-1), \\
& f(n, j)=0 \quad \text { if } j>t+m-r-1 . \\
& f(n, j)=2^{m-j}+2^{m-j+2}+\cdots+2^{m-j+2(j-1)} \\
& (j=1, \cdots, t), \\
& f(n, t+j)=2^{m+t-(j+2)}+2^{m+t-(j+4)}+\cdots+2^{m+t-(j+2 t)} \\
& (j=1, \cdots, m-r-t-1), \\
& f(n, m-r+j)=2^{r+j}+2^{r+j+2}+\cdots+2^{r+j+2(t-j-1)} \\
& (j=0, \cdots, t-1), \\
& f(n, j)=0 \quad \text { if } j>t+m-r-1 .
\end{aligned}
$$

For example, if $n=2^{t}$, then

$$
f(n, 0)=2, \quad f(n, j)=2^{j-1} \quad(0<j \leqq t) .
$$

THEOREM 3.2. If (1.1) holds, and $e_{1}=u>0, e_{i}-e_{i-1}=1$ for $i=2$, . . , m-1, $e_{m}-e_{m-1}=2, m-1 \geqq u$, then

$$
\begin{aligned}
& f(n, 0)=2^{m} \text {, } \\
& f(n, 1)=2^{m} \text {, } \\
& f(n, j)=2^{m+j-2}+2 \cdot 2^{m+j-4}+\cdots+2 \cdot 2^{m+j-2 j} \\
& (j=2, \cdots, u), \\
& f(n, u+j)=2^{m+u-j-1}+2^{m+u-j-8}+\cdots+2^{m+u-j-(2 u-1)} \\
& (j=1, \cdots, m-u-1), \\
& f(n, m)=1+2 \cdot 2^{2}+\cdots+2 \cdot 2^{2(u-1)}, \\
& f(n, m+j)=2^{j+1}+2^{j+3}+\cdots+2^{j+2(u-j)-1} \\
& (j=1, \cdots, u-2), \\
& f(n, u+m-1)=2^{u} \quad(\text { if } u>1), \\
& f(n, u+m)=2^{u} . \\
& f(n, j)=2^{m+j-2}+2 \cdot 2^{m+j-4}+\cdots+2 \cdot 2^{m+j-2 j} \\
& (j=2, \cdots, m-1) \text {, }
\end{aligned}
$$

If $m-1<u$, then 


$$
\begin{aligned}
f(n, m) & =1+2 \cdot 2^{2}+\cdots+2 \cdot 2^{2(m-2)}+2^{2 m-2}, \\
f(n, m+j) & =2^{2 m+j-2}+2 \cdot 2^{2 m+j-4}+\cdots+2 \cdot 2^{2 m+j-2 m} \\
f(n, u+j) & =2^{m+u-j-1}+2^{m+u-j-3}+\cdots+2^{u+m-j-(2 m-2 j-1)} \\
& \\
f(n, u+m-1) & =2^{u} \quad(j=1, \cdots, u-m), \\
f(n, u+m) & =2^{u} .
\end{aligned}
$$

In all of the following theorems, we assume that (1.1) holds and $f(n, j)$ is given by (2.2). Theorems 3.3 and 3.4 are proved in [3].

THEOREM 3.3. If $e_{i}-e_{i-1}>j$ for $q$ terms $e_{i}$ and $e_{i}-e_{i-1}=j$ for $m-q$ terms $e_{i}$, then

$$
\begin{aligned}
& a_{j 1}=q, \\
& a_{j i}=\left(\begin{array}{c}
m \\
i
\end{array}\right)\left(\begin{array}{l}
j-1 \\
i-1
\end{array}\right) \quad(i=2, \cdots, j) .
\end{aligned}
$$

THEOREM 3.4. If $e_{i}-e_{i-1}>j$ for $q$ terms $e_{i}, e_{i}-e_{i-1}=j$ for $m-q-1$ terms $e_{i}$, and $e_{1}=u, 0 \leqq u<j-1$, then

$$
\begin{aligned}
a_{j 1}=q, \quad a_{j i}=\left(\begin{array}{c}
m \\
i
\end{array}\right)\left(\begin{array}{c}
j-1 \\
i-1
\end{array}\right)-\left(\begin{array}{c}
m-1 \\
i-1
\end{array}\right)\left(\begin{array}{c}
j-u-1 \\
i-1
\end{array}\right) \\
(i=2, \cdots, j) .
\end{aligned}
$$

To get more results of this type, we use the method described in $\S 2$. We also use the fact that the number of compositions of $j$ into $i$ parts, $j=x_{1}+x_{2}+\cdots+x_{i}$, such that $x_{1}>u_{1}, x_{2}>u_{2}, \cdots, x_{k}>u_{k}$ is

$$
\left(\begin{array}{c}
j-u_{1}-\cdots-u_{k}-1 \\
i-1
\end{array}\right)
$$

See $[4$, p. 124] and [3].

THEOREM 3.5. If $e_{i}-e_{i-1}>j$ for $q$ terms $e_{i}, e_{i}-e_{i-1}=j$ for $m-q-1$ terms $e_{i}$ and $e_{k}-e_{k-1}=u<j$ for some $k \neq 1$, then

$$
\begin{aligned}
& a_{j 1}=q, \\
& a_{j i}=\left(\begin{array}{c}
m \\
i
\end{array}\right)\left(\begin{array}{c}
j-1 \\
i-1
\end{array}\right)-\left(\begin{array}{c}
j-u-1 \\
i-1
\end{array}\right)\left(\begin{array}{c}
m-2 \\
i-2
\end{array}\right)-\left(\begin{array}{c}
m-2 \\
i-1
\end{array}\right)\left(\begin{array}{c}
j-u \\
i-1
\end{array}\right) \\
&(i=2, \cdots, j) .
\end{aligned}
$$


TheOREM 3.6. If $e_{i}-e_{i-1}>j$ for $q$ terms $e_{i}, e_{i}-e_{i-1}=j$ for $m-q-2$ terms $e_{i}$ and $e_{1}=v<j-1, e_{k}-e_{k+1}=u<j$ for some $k>2$, then

$$
\begin{aligned}
a_{j 1}= & q, \\
a_{j i}= & \left(\begin{array}{c}
m \\
i
\end{array}\right)\left(\begin{array}{l}
j-1 \\
i-1
\end{array}\right)-\left(\begin{array}{c}
j-u-1 \\
i-1
\end{array}\right)\left(\begin{array}{c}
m-2 \\
i-2
\end{array}\right) \\
& -\left(\begin{array}{c}
m-2 \\
i-1
\end{array}\right)\left(\begin{array}{c}
j-u \\
i-1
\end{array}\right)-\left(\begin{array}{c}
j-v-1 \\
i-1
\end{array}\right)\left(\begin{array}{c}
m-1 \\
i-1
\end{array}\right) \\
& +\left(\begin{array}{c}
j-u-v \\
i-1
\end{array}\right)\left(\begin{array}{c}
m-3 \\
i-2
\end{array}\right)+\left(\begin{array}{c}
j-u-v-1 \\
i-1
\end{array}\right)\left(\begin{array}{c}
m-3 \\
i-3
\end{array}\right) \\
& (i=2, \cdots, j) .
\end{aligned}
$$

TheOREM 3.7. If $e_{i}-e_{i-1}>j$ for $q$ terms $e_{i}, e_{i}-e_{i-1}=j$ for $m-q-2$ terms $e_{i}, e_{2}-e_{1}=u<j, e_{1}=v<j-1$, then

$$
\begin{aligned}
& a_{j 1}= q, \\
& a_{j i}=\left(\begin{array}{c}
m \\
i
\end{array}\right)\left(\begin{array}{l}
j-1 \\
i-1
\end{array}\right)-\left(\begin{array}{c}
m-2 \\
i-1
\end{array}\right)\left(\begin{array}{l}
j-u \\
i-1
\end{array}\right)-\left(\begin{array}{c}
m-2 \\
i-2
\end{array}\right)\left(\begin{array}{c}
j-u-1 \\
i-1
\end{array}\right) \\
&-\left(\begin{array}{c}
m-1 \\
i-1
\end{array}\right)\left(\begin{array}{c}
j-v-1 \\
i-1
\end{array}\right)+\left(\begin{array}{c}
m-2 \\
i-2
\end{array}\right)\left(\begin{array}{c}
j-u-v-1 \\
i-1
\end{array}\right) \\
&(i=2, \cdots, j) .
\end{aligned}
$$

THEOREM 3.8. If $e_{i}-e_{i-1}>j$ for $q$ terms $e_{i}, e_{i}-e_{i-1}=j$ for $m-q-2$ terms $e_{i}, e_{k+1}-e_{k}=u<j, e_{k}-e_{k-1}=v<j$ for some $k>1$, then

$$
\begin{aligned}
a_{j 1}= & q, \\
a_{j i}= & \left(\begin{array}{c}
m \\
i
\end{array}\right)\left(\begin{array}{l}
j-1 \\
i-1
\end{array}\right)-\left(\begin{array}{c}
m-2 \\
i-2
\end{array}\right)\left(\left(\begin{array}{c}
j-u-1 \\
i-1
\end{array}\right)+\left(\begin{array}{c}
j-v-1 \\
i-1
\end{array}\right)\right) \\
& -\left(\begin{array}{c}
m-2 \\
i-1
\end{array}\right)\left(\left(\begin{array}{c}
j-u \\
i-1
\end{array}\right)+\left(\begin{array}{l}
j-v \\
i-1
\end{array}\right)\right) \\
& +\left(\begin{array}{c}
j-u-v-1 \\
i-1
\end{array}\right)\left(\begin{array}{c}
m-3 \\
i-3
\end{array}\right)+\left(\begin{array}{c}
j-u-v \\
i-1
\end{array}\right)\left(\begin{array}{c}
m-3 \\
i-2
\end{array}\right) \\
& (i=2, \cdots, j) .
\end{aligned}
$$

THEOREM 3.9. If $e_{i}-e_{i-1}>j$ for $q$ terms $e_{i}, e_{i}-e_{i-1}=j$ for $m-q-2$ terms $e_{i}, e_{k}-e_{k-1}=u<j$ for some $k>1, e_{t}-e_{t-1}=v<j$ for some $t>k+1$, then 


$$
\begin{aligned}
a_{j 1}= & q, \\
a_{j i}= & \left(\begin{array}{c}
m \\
i
\end{array}\right)\left(\begin{array}{l}
j-1 \\
i-1
\end{array}\right)-\left(\begin{array}{c}
j-u-1 \\
i-1
\end{array}\right)\left(\begin{array}{c}
m-2 \\
i-2
\end{array}\right)-\left(\begin{array}{l}
j-u \\
i-1
\end{array}\right)\left(\begin{array}{c}
m-2 \\
i-1
\end{array}\right) \\
& -\left(\begin{array}{c}
j-v-1 \\
i-1
\end{array}\right)\left(\begin{array}{c}
m-2 \\
i-2
\end{array}\right)-\left(\begin{array}{l}
j-v \\
i-1
\end{array}\right)\left(\begin{array}{c}
m-2 \\
i-1
\end{array}\right) \\
& +\left(\begin{array}{c}
j-u-v+1 \\
i-1
\end{array}\right)\left(\begin{array}{c}
m-4 \\
i-2
\end{array}\right)+2\left(\begin{array}{c}
j-u-v \\
i-1
\end{array}\right)\left(\begin{array}{c}
m-4 \\
i-3
\end{array}\right) \\
& +\left(\begin{array}{c}
j-u-v-1 \\
i-1
\end{array}\right)\left(\begin{array}{c}
m-4 \\
i-4
\end{array}\right) \quad(i=2, \cdots, j) .
\end{aligned}
$$

For example, again consider $n=2^{4}+2^{5}+2^{20}+2^{26}+2^{28}$ and suppose we wish to find $f(n, 4)$. Using Theorem 3.9 , we have $j=4, q=3, u=1$, $v=2, m=5$, and so

$$
f(n, 4)=3 \cdot 2^{7}+14 \cdot 2^{5}+16 \cdot 2^{3}+4 \cdot 2 .
$$

Finally, we determine the positive integer $s$ such that $f(n, s) \neq 0$ and $f(n, j)=0$ for $j>s$. Suppose

$$
e_{i}-e_{i-1} \leqq k \quad(i=1, \cdots, m)
$$

and suppose

$$
e_{i}-e_{i-1}=1 \quad(i=1, \cdots, r), \quad e_{r+1}-e_{r}>1 .
$$

We note that $r=0$ if $e_{1}>0$. If for $i>r, e_{i}-e_{i-1}=t$ for $h_{t}$ terms $e_{i}$ $(t=1, \cdots, k)$, then it is clear by the remarks of $\$ 2$ that

$$
f(n, j)=0 \quad \text { if } j>h_{1}+2 h_{2}+\cdots+k h_{k}-1=e_{m}-r
$$

and $f\left(n, e^{m}-r\right)=2^{h_{2}+2 h_{3}+\cdots+(k-1) h_{k}+r-1}$.

For example, if $n=2^{4}+2^{5}+2^{20}+2^{26}+2^{28}$ then $r=0, h_{1}=h_{2}=h_{5}$ $=h_{6}=h_{15}=1$ and $f(n, j)=0$ if $j>28, f(n, 28)=2^{23}$.

\section{REFERENCES}

1. L. Carlitz, The number of binomial coefficients divisible by a fixed power of a prime, Rend. Circ. Mat. Palermo (2) 16 (1967), 299-320. MR 40 \#2554.

2. L. E. Dickson, History of the theory of numbers. Vol. 1, reprint, Chelsea, New York, 1952.

3. F. T. Howard, A combinatorial problem and congruences for the Rayleigh function, Proc. Amer. Math. Soc. 26 (1970), 574-578.

4. J. Riordan, An introduction to combinatorial analysis, Wiley, New York; Chapman \& Hall, London, 1958. MR 20 \#3077.

Wake Forest University, Winston-Salem, North Carolina 27109 